\title{
Pectina: da Matéria-Prima ao Produto Final
}

\author{
Maria H. G. Canteri \\ Coordenação de Alimentos, UTFPR \\ Lirian Moreno, Gilvan Wosiacki \\ Departamento de Engenharia de Alimentos, UEPG \\ Agnes de P. Scheer \\ Setor de Tecnologia, UFPR
}

\begin{abstract}
Resumo: A pectina, provavelmente a mais complexa macromolécula natural, é um heteropolissacarídeo contendo predominantemente resíduos de ácido galacturônico. Este polímero, do grupo das fibras dietéticas, é amplamente utilizado como geleificante e estabilizante na indústria de alimentos. O principal processo industrial para obtenção de pectina está baseado na solubilização da protopectina do bagaço de maçã e casca de frutos cítricos, realizada em condições levemente ácidas sob aquecimento. Estudos recentes têm reportado a extração de pectina de novas matérias-primas sob diferentes condições, com influência sobre a qualidade e sobre o rendimento do produto final, para aumentar sua qualidade reológica. Esta revisão descreve a estrutura, as fontes, as aplicações, o processo de extração industrial assim como as principais técnicas de caracterização da pectina.
\end{abstract}

Palavras-chave: Pectina, extração, matéria-prima, estrutura, qualidade.

\section{Pectin: from Raw Material to the Final Product}

Abstract: Pectin, probably the most complex macromolecule in nature is a hetero-polysaccharide containing predominantly residues of galacturonic acid (GalA). This polymer, which belongs to a group of dietary fibers, is widely used as a gelling agent and stabilizer in the food industry. The main industrial processing to obtain pectin is based on the solubilization of protopectin from apple pomace and citrus peels, which is done under low acidity and heated conditions. Recent studies have reported the extraction of pectin from new raw materials and using different extraction conditions, which influence the yield and quality of the final product, and may improve the rheological properties. This review describes the structure, sources, applications and industrial extraction processes, as well as the analysis methods of physicochemical characterization of pectin.

Keywords: Pectin, extraction, raw material, structure, quality.

\section{Introdução}

A pectina refere-se a uma família de oligossacarídeos e polissacarídeos, com características comuns, todavia extremamente diversos em sua estrutura fina. $\mathrm{O}$ esqueleto péctico é primariamente um homopolímero de ácido galacturônico ligado em $\alpha(1 \rightarrow 4)$, com grau variável de grupos carboxilas metil esterificados ${ }^{[1-4]}$. A pectina deve ser constituída de, no mínimo, $65 \%$ de ácido galacturônico, segundo a FAO (Organização das Nações Unidas para a Alimentação e Agricultura) e EU (União

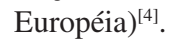

Devido à grande variedade de matérias-primas existem também grandes diferenças no poder geleificante de preparações de pectina ${ }^{[1]}$. A pectina comercial em pó pode ser classificada como de alta metoxilação (HM ou AM), com percentual de grupamentos esterificados na cadeia (grau de esterificação ou DE) superior a $50 \%$, porém na prática apresenta-se entre 50 e $75 \%$, ou de baixa metoxilação (LM ou BM), com DE inferior a 50\%, na prática entre 20 e $45 \%{ }^{[3,5,6]}$.

Em função da pectina apresentar-se como alternativa para agregação de valores aos resíduos sólidos vegetais, associado à minimização do volume a ser descartado, este trabalho possibilitou o acesso à informações relacionadas à extração e caracterização de substâncias pécticas, indicando diretrizes futuras para pesquisas com relação a esse polímero.

\section{Histórico}

As primeiras citações sobre pectina datam de um artigo inglês de 1750 sobre preparação de geleia de maçãs ${ }^{[7]}$. A descoberta da pectina, enquanto composto químico, foi feita por Vauquelin em 1790 e Braconnot, no ano de 1824, foi o primeiro a caracterizála como composto das frutas responsável pela formação do gel e sugerir o nome pectina, proveniente do grego $\pi \eta \chi \tau o \zeta$, que significa espesso ${ }^{[8]}$. A ocorrência de substâncias pécticas diferindo em solubilidade e facilidade de extração é conhecida desde 1848, quando Fremy reportou a existência de um precursor péctico insolúvel em água, denominado posteriormente de protopectina, por Tschirch ${ }^{[9]}$. Durante a investigação da turbidez de suco, Kelhofer, em 1908, concluiu que o sedimento observado em fermentado de pera compreendia um complexo de proteínas, pectinas e compostos fenólicos oxidados ${ }^{[10]}$. Até meados de 1930 , a pectina era considerada como uma pequena estrutura cíclica. Smolenski, em 1923, foi o primeiro a sugerir que a pectina era um polímero complexo, comparável em estrutura ao amido, 
sendo que as análises de raios-X concordavam com essa hipótese, mas indicavam ser mais lógica a comparação com a celulose ${ }^{[11]}$. De acordo com Meyer e Mark (1930 apud Baker), os ácidos pectínicos apresentam grupos metiléster ligados em $\alpha(1 \rightarrow 4)^{[12]}$ e a fórmula básica foi estabelecida por Schneider e Bock, em 1937 ${ }^{[13]}$. Nessa época, já se discutia a dificuldade em estabelecer estudos comparativos quanto à composição, ao tipo e à distribuição dos compostos pécticos em vegetais, devido aos diferentes métodos de extração e análises empregados ${ }^{[14]}$.

\section{Produção de pectina}

\section{Histórico da produção comercial}

A obtenção de um extrato líquido de pectina foi registrada em 1908 na Alemanha e o processo propagou-se rapidamente pelos Estados Unidos, onde uma patente clássica foi obtida por Douglas (US Patente n. 1.082,682 de 1913) ${ }^{[15]}$. A produção de pectina desenvolveu-se aos poucos, no início do século XX, na Europa e nos Estados Unidos, usando principalmente o bagaço de maçã. A primeira fábrica de pectina cítrica foi construída em 1926, na Califórnia ${ }^{[7]}$.

Quanto à produção comercial, Hermann Herbsthreith descobriu, na década de 1930, o potencial de uso e aplicação do bagaço de maçã, subproduto até então descartado da produção do suco ${ }^{[16]}$. Em 1940, cerca de $60 \%$ da pectina produzida no mundo era de bagaço cítrico, devido à abundância de matéria-prima gerada pela industrialização de $\operatorname{sucos}^{[7]}$.

A produção industrial brasileira teve início no ano de 1954, na cidade de Limeira-SP ${ }^{[17]}$, sendo a única fábrica de pectina ainda hoje no País e maior produtora mundial de pectina cítrica ${ }^{[18]}$.

Recentemente, foram desenvolvidos estudos sobre potencial de uso do bagaço de maçã na recuperação de compostos fenólicos nativos, associados ao escurecimento, para utilização como agentes antioxidantes, resultando na coloração mais clara da pectina obtida $^{[19]}$. Isso aponta a tendência crescente das indústrias em buscar alternativas que promovam o fechamento da "reciclagem" de um resíduo, com o máximo de aproveitamento e por meio da maior exploração comercial dos componentes antes considerados como subprodutos.

Na maioria dos países em vias de desenvolvimento, a pectina é importada. A produção regionalizada por pequenos processadores poderia suprir a demanda com custo mais baixo, desde que existisse incentivo governamental. Entretanto, existem algumas barreiras a serem superadas, como o custo, a dificuldade para isolamento da pectina sob a forma de pó em pequena escala e a obtenção de uma gama de pectinas com propriedades específicas, e não apenas de um tipo de produto. Tipicamente, a produção de pectina nos países tropicais em desenvolvimento está coimplantada junto às indústrias de suco em larga escala. Poucos fabricantes detêm o comércio global e curiosamente, toda pectina utilizada nos EUA é importada, principalmente da Europa e das Américas Central e do Sul ${ }^{[20,21]}$.

As principais indústrias mundiais produtoras de pectina, na atualidade, são Cargill, Cítrico, CPkelco, Danisco, Degussa, Herbestreith \& Fox, Obipectin e Pektowin Jaslo ${ }^{[22]}$. O valor de venda de um quilograma de pectina cítrica de alta metoxilação no Brasil (janeiro de 2011) indica valorização de mais de 500\% em cerca de vinte anos ${ }^{[23,24]}$.

\section{Produção industrial}

Embora a extração de pectina varie de acordo com a matériaprima, em linhas gerais, o processo compreende: extração do vegetal de origem em meio aquoso ácido; purificação desse líquido extraído e isolamento da pectina por precipitação ${ }^{[25]}$. A maior parte da pectina solúvel em água permanece no suco e a remanescente é insolúvel. A solubilização dessa fração menos solúvel envolve processos físicos e químicos, sendo acompanhada por inevitável e não controlada remoção de açúcares neutros da cadeia lateral, bem como pela hidrólise de ligações ésteres ${ }^{[1]}$.

A extração em meio ácido sob aquecimento é o método utilizado industrialmente para obtenção de pectinas a partir de resíduos industriais de sucos de frutas ${ }^{[26]}$. Diferentes ácidos podem ser utilizados nesse processo. Em alguns países, os ácidos minerais são proibidos, sendo substituídos por cítrico, lático ou tartárico ${ }^{[27]}$. As condições são variáveis, mas via de regra um $\mathrm{pH}$ na faixa de 1,5-3,0 é utilizado por 0,5-6,0 horas, numa faixa de temperatura de 60-100 ${ }^{\circ} \mathrm{C}$. A razão sólido-líquido é geralmente $1: 18$, sendo cerca de 1:15 para o bagaço de maçã e 1:35 para o bagaço cítrico, ambos desidratados $^{[1,27]}$.

Industrialmente, a pectina extraída é separada do bagaço utilizando prensas hidráulicas e/ou centrifugação. Algumas vezes, ocorre gelatinização do amido e é necessário tratamento enzimático preliminar com amilases. Subsequentemente, o extrato é filtrado e, finalmente, concentrado. Na preparação de pectinas em pó, o extrato líquido concentrado é tratado com solventes orgânicos ou certos sais metálicos para precipitar os polímeros ${ }^{[27]}$.

A pectina precipita em concentrações de etanol mais elevadas que $45 \%(\mathrm{~m} / \mathrm{v})$. Metanol, etanol e 2-propanol podem ser usados. Para minimizar o volume de álcool, o extrato clarificado pode ser concentrado em evaporadores multiestágio até 3-4\% de teor de pectina. O precipitado obtido pela adição de etanol é lavado posteriormente para remover contaminantes na forma de metais pesados, resíduos de agrotóxicos, ácidos, açúcares, compostos fenólicos, pigmentos e outros materiais insolúveis em álcool ${ }^{[1]}$. A pectina precipitada é coletada, seca e moída ${ }^{[27]}$.

De uma forma geral, no armazenamento, mesmo pulverizadas, as pectinas sofrem algumas despolimerizações e desmetilações, num processo de auto-hidrólise, mesmo na forma pulverizada, principalmente se a pectina estiver na forma ácida e a taxa de umidade acima de $5 \%$. O pH de estabilidade situa-se entre 3,5 e $4,5^{[26]}$.

Pectinas com rápida geleificação, com grau de metoxilação maior que $70 \%$ (DM>70\%), são tipicamente extraídas em $\mathrm{pH} 2,5$ e $100{ }^{\circ} \mathrm{C}$ por 45 minutos. Pectinas com velocidade média ou lenta de geleificação (DM 60-70\%) são extraídas em temperaturas mais baixas por períodos mais longos de tempo (por exemplo, $60{ }^{\circ} \mathrm{C}$, 4 horas), visto que em baixas temperaturas o procedimento de desesterificação é mais rápido que a despolimerização. $\mathrm{O}$ extrato obtido tipicamente contém entre 0,3 e $0,5 \%$ de pectina ${ }^{[1]}$.

As pectinas comerciais são produtos padronizados com sacarose, glucose ou lactose até grau de geleificação de $150{ }^{\circ}$ US SAG, para assegurar ao usuário a mesma força de gel ${ }^{[1,27]}$. A padronização pode modificar a estrutura química das pectinas, promovendo a saponificação parcial de ésteres ou a amidação de grupamentos ácidos $^{[27]}$.

\section{Fontes}

O teor em substâncias pécticas varia de acordo com a origem botânica do produto vegetal, sendo quatro subprodutos de indústrias agrícolas e alimentares ricos em substâncias pécticas (teor superior a $15 \%$ em base seca): bagaço de maçã, albedo cítrico, polpa de beterraba e capítulos de girassol ${ }^{[28]}$. A maior parte da pectina usada pela indústria de alimentos é originária dessas matérias-primas, sendo extraída em condições levemente ácidas e em alta temperatura, resultando primariamente em cadeias de homogalacturonana ${ }^{[4]}$. Pesquisas com fontes convencionais cada 
vez mais são aprofundadas, com relação à extração e aos propósitos de elucidar aspectos diversos: tecnológicos ou estruturais ${ }^{[29-38]}$.

Devido à capacidade de geleificação depender da massa molar e grau de esterificação, pectinas de diferentes fontes não apresentam a mesma habilidade de formação de gel em função das variações nestes parâmetros. Em consequência, a detecção de uma larga quantidade de pectina em uma fruta não é suficiente para qualificála como fonte comercial de pectina. Novas fontes potenciais permanecem não usuais em função de certas propriedades estruturais indesejáveis ${ }^{[3,39]}$. Dentre as matérias-primas alternativas estudadas para extração de substâncias pécticas podem ser citadas: os frutos de Chaemomeles japonica ou marmelo do Japão ${ }^{[39]}$, as folhas do Krueo Ma Noy, uma planta trepadeira nativa da Tailândia ${ }^{[40,41]}$, o pericarpo de ambarela (fruta nativa da Polinésia) ${ }^{[42]}$, a casca de manga ${ }^{[43,44-45]}$, o repolho ${ }^{[46]}$, as cascas de banana ${ }^{[47]}$, o resíduo de cacau $^{[48]}$ e a casca de maracujá ${ }^{[49-54]}$.

$\mathrm{Na}$ Tabela 1, podem ser encontrados os teores aproximados de pectina em diferentes fontes vegetais ${ }^{[55,56]}$.

\section{Aspectos Fisiológicos da Pectina}

\section{Localização}

A pectina está localizada na parede celular de vegetais superiores associada a outros componentes. Essa parede pode ser dividida em duas camadas denominadas de primária e secundária. Dois modelos estruturais são propostos para a parede celular primária, cuja área de superfície aumenta durante a expansão da célula: tipo I, composto principalmente por celulose, xiloglucanas, extensina e pectina (20-35\%) e tipo II, compreendendo essencialmente celulose, glucoarabinoxilanas e compostos fenólicos e menor proporção de pectina (10\%). A parede secundária, estrutura complexa adaptada à função da célula, é formada internamente à parede primária na diferenciação ${ }^{[57,58]}$.

Entre as paredes primárias de células vizinhas encontra-se uma estrutura denominada lamela média, formada por moléculas

Tabela 1. Conteúdo de pectina de alguns frutos ${ }^{[5,56]}$.

\begin{tabular}{|c|c|c|}
\hline Fruto & $\%$ em MF & $\%$ em MS \\
\hline Maçã (Malus sp.) & $0,5-1,6$ & $4-7$ \\
\hline Bagaço de maçã & $1,5-2,5$ & $15-20$ \\
\hline Albedo cítrico (Citrus sp.) & - & $30-35$ \\
\hline Casca de laranja (Citrus sinensis) & $3,5-5,5$ & - \\
\hline Maracujá (Passiflora edulis S.) & 0,5 & - \\
\hline Maracujá gigante (Passiflora quadrangularis L.) & 0,4 & - \\
\hline Casca de maracujá & $2,1-3,0$ & - \\
\hline Batata & - & 2,5 \\
\hline Banana (Musa acuminata) & $0,7-1,2$ & - \\
\hline Beterraba (Beta vulgaris) & 1,0 & - \\
\hline Bagaço de beterraba & - & $15-20$ \\
\hline Carambola (Averrhoa carambola) & 0,7 & - \\
\hline Cenoura (Daucus carota) & $0,2-0,5$ & 10 \\
\hline Goiaba (Psidium guajava) & $0,8-1,0$ & - \\
\hline Polpa de limão (Citrus lemon) & $2,5-4,0$ & - \\
\hline Lichia (Litchi chinensis S.) & 0,4 & - \\
\hline Manga (Mangifera indica L.) & $0,2-0,4$ & - \\
\hline Mamão (Carica papaya) & $0,7-1,0$ & - \\
\hline Pêssegos (Prunus persica) & $0,1-0,9$ & - \\
\hline Abacaxi (Ananas comosus L.) & $0,04-0.1$ & - \\
\hline Morangos (Fragaria ananassa) & $0,6-0,7$ & - \\
\hline Tamarindo (Tamarindus indica L.) & 1,71 & - \\
\hline Tomate (Lycopersicon esculentum) & $0,2-0,6$ & 3 \\
\hline
\end{tabular}

$\mathrm{MF}=$ material-fresca; $\mathrm{MS}$ = material-seca. de pectina, provavelmente de alta metoxilação, unidas por meio de ligações cruzadas, com camadas de cadeias de homogalacturonana depositadas subsequentemente em pectinas de células opostas ${ }^{[58,59]}$.

Os fatores que envolvem a deposição de pectina durante a formação da parede celular ainda não estão elucidados. Os polissacarídeos pécticos parecem ser importantes no controle da porosidade da parede, na adesão de células subjacentes e no controle do ambiente iônico da parede celular. Análises de mutações que alteram a estrutura de uma das cadeias de pectina, a ramnogalacturonana II, indicam que alguns tipos de ligações pécticas tem importância também sobre o reforço da parede, a aderência intercelular e o crescimento normal em plantas vasculares ${ }^{[60]}$. Outra função está ligada aos mecanismos de defesa vegetal. O ácido galacturônico, unidade básica da pectina, é um componente essencial de elicitores ativos da parede celular, substâncias que provocam o acúmulo de fitoalexinas com propriedades antibióticas nos locais de infecção vegetal ${ }^{[61]}$.

\section{Transformações pécticas ocorridas durante o amadurecimento de frutos}

Estudos sobre o metabolismo de parede celular durante o amadurecimento indicam ser um processo ordenado, com relaxamento da parede celular e degradação de hemicelulose nos estádios iniciais seguida pela despolimerização da pectina, ao final. O aumento da solubilidade dos polissacarídeos pécticos é uma das características indiscutivelmente associadas ao amadurecimento de frutas frescas. Os mecanismos que contribuem nesse processo não estão completamente esclarecidos e sua magnitude varia acentuadamente entre diferentes frutas. Os radicais livres produzidos por meios enzimáticos ou químicos podem participar da hidrólise de pectinas ${ }^{[62]}$. Oligogalacturonídeos, moléculas lineares com duas a vinte unidades de ácido galacturônico liberados a partir da fragmentação da homogalacturonana da parede celular primária, estão envolvidos no amadurecimento dos frutos induzindo a produção de etileno. Esse fenômeno foi estudado em tomate e frutos cítricos e essa função parece ser bastante complexa ${ }^{[63]}$.

\section{Controle de qualidade de frutos e produtos derivados}

As substâncias pécticas de vários frutos nativos do Brasil, ou de variedades brasileiras de certos frutos utilizados na elaboração de doces e geleias, têm sido amplamente exploradas, com o objetivo de avaliar o potencial tecnológico da matéria-prima e/ou permitir o controle de qualidade no desenvolvimento de produtos derivados. Estudos de pesquisadores do Brasil com resultados relacionados à pectina foram conduzidos na gabiroba ${ }^{[64-66]}$, amorapreta $^{[67,68]}$, morango e framboesa ${ }^{[68]}$, pequi ${ }^{[69]}$, abacaxi ${ }^{[70]}$, casca de $\operatorname{maracujaj}^{[54,71,72]}$, goiaba ${ }^{[73]}$, maçã $\tilde{a}^{[33,34,38,74-76]}$.

\section{Estrutura molecular}

Em 1934, as pectinas cítricas foram reconhecidas como cadeias lineares de ácido galacturônico e desde então, evidenciou-se que a pectina é uma molécula altamente complexa e o recente grande desafio é acomodar toda informação disponível em um único modelo estrutural $^{[4]}$. As pectinas são formadas por dezessete diferentes monossacarídeos, organizados em polissacarídeos distintos, a partir de mais de vinte diferentes ligações para formação de uma rede quando unidos ${ }^{[4,59]}$, agrupados em diferentes tipos de cadeia, constituídas por ácidos urônicos, hexoses, pentoses e metilpentoses. Diversas unidades estruturais podem estar substituídas por metanol, ácido acético e ácidos fenólicos. Os açúcares podem existir nas formas furanosídicas ou piranosídicas e com diferentes anômeros 
( $\alpha$ ou $\beta$ ), com diversos tipos de ligações entre os monômeros, tais como $\alpha(1 \rightarrow 4), \alpha(1 \rightarrow 5), \beta(1 \rightarrow 3)$ e $\beta(1 \rightarrow 4)$ e $\beta(1 \rightarrow 6)^{[26]}$.

A homogalacturonana (HG) é o mais abundante polissacarídeo péctico na parede celular, correspondente a cerca de 60-65\% do total da pectina ${ }^{[4,58]}$. Apresenta unidades de ácido $\alpha-D-$ galactopiranosilurônico em ligações $1 \rightarrow 4$ num padrão linear. Os grupos carboxilas estão parcialmente metil-esterificados. As cadeias podem ser, em função da fonte vegetal, parcialmente O-acetiladas no $\mathrm{C}-3$ ou C-2 $2^{[3,58,59]}$.

A ramnogalacturonana I (RG-I) apresenta uma cadeia representada pelo dissacarídeo $[\rightarrow 4-\alpha-\mathrm{D}-\mathrm{GalA}-(1 \rightarrow 2)-\alpha-\mathrm{L}-$ Rha- $(1 \rightarrow] \mathrm{n}^{[3,4,58,59]}$. Em resumo, uma variedade de diferentes cadeias de glucanas (principalmente arabinana e galactana) está ligada às unidades de ramnose. $\mathrm{O}$ comprimento das cadeias pode variar consideravelmente e a composição de açúcares de RG-I pode ser altamente heterogênea ${ }^{[3]}$. A RG-I representa 20-35\% da pectina, com alto grau de especialização celular e expressão dependente do desenvolvimento, no tipo e número de açúcares simples e oligossacarídeos ligados a essa cadeia. A razão para esse nível de variação em RG-I não é conhecida, mas sugere diversidade funcional. A ramnogalacturonana II (RG-II) é o segmento estruturalmente mais complexo e compõe 10\% da pectina. Essa estrutura, altamente conservada na maior parte das espécies vegetais, consiste de um esqueleto de homogalacturonana de no mínimo oito (provavelmente mais) unidades monoméricas, contendo cadeias laterais de até 12 diferentes tipos de açúcares, alguns muito peculiares como apiose, ácido acérico, ácido 3-deoxi-lixo-2-heptulosárico (DHA) e ácido 3 deoxi-mano-2-octulosônico (KDO). A RG-II usualmente existe em paredes celulares como dímeros em ligações cruzadas por um borato diol éster entre unidades apiosil na cadeia latera ${ }^{[3,4]}$.
A xilogalacturonana (XGA) é uma homogalacturonana substituída com xilose ligada na posição 3 . O grau de xilosidação pode variar entre $25 \%$ (melancia) a $75 \%$ (maçã). Essa xilose pode ser adicionalmente substituída em O-4 com outra xilose em ligação $\beta$, sendo mais prevalente em tecidos reprodutivos como frutas e sementes ${ }^{[4,58]}$.

A arabinogalactana I (ARA-I) é composta por esqueleto $\beta$-D-Gal $p$; resíduos de $\alpha$-L-Araf podem estar ligados às unidades galactosil na posição 3. A arabinogalactana II (ARA-II) está principalmente associada com proteínas (3-8\%), também denominadas arabinogalactanas-proteínas (AGPs). A AG-II é composta de um esqueleto $\beta$-D-Gal $p \quad 1 \rightarrow 3$, contendo curtas cadeias de $[\alpha$-L-Araf $(1 \rightarrow 6) \beta$-D-Galp $(1 \rightarrow 6)] n$ onde $n=1,2$ ou 3. A parte proteica é rica em prolina, hidroxiprolina, alanina, serina e treonina ${ }^{[4]}$. A arabinana (ARA) consiste de um esqueleto de $\alpha$-L-Araf em ligações $1 \rightarrow 5$, onde podem estar cadeias laterais de $\alpha$-L-Ara $f(1 \rightarrow 2)$ e $\alpha$-L-Ara $f(1 \rightarrow 3)$ e/ou cadeias laterais de $\alpha$-L-Araf $(1 \rightarrow 3) \alpha$-L-Araf $(1 \rightarrow 3)^{[55]}$. Existe também outra cadeia não representada esquematicamente, a apiogalacturonana (API), HG substituída em O-2 ou O-3 com D-apiofuranose. Está presente em monocotiledôneas aquáticas como Lemna ${ }^{[58]}$.

Estudos permitiram esquematizar estruturas hipotéticas da pectina, com breves modificações de acordo com novas evidências analíticas. Na representação esquemática de Willats, Knox e Mikkelsen ${ }^{[3]}$ está indicada a alternativa de modelo proposta a partir da representação convencional anteriormente mais usual (Figura 1).

\section{Degradação da pectina}

Em dispersão, as substâncias pécticas podem degradar-se por dois mecanismos químicos principais, esquematizados em três tipos

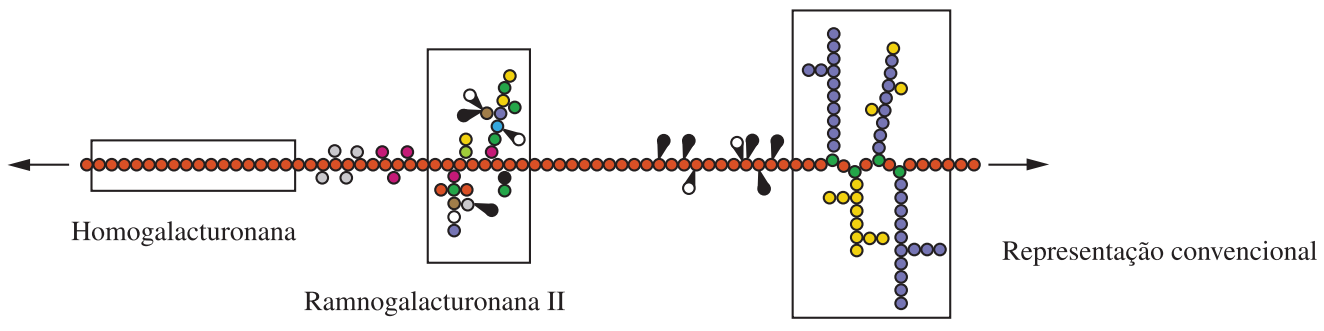

Ramnogalacturonana I

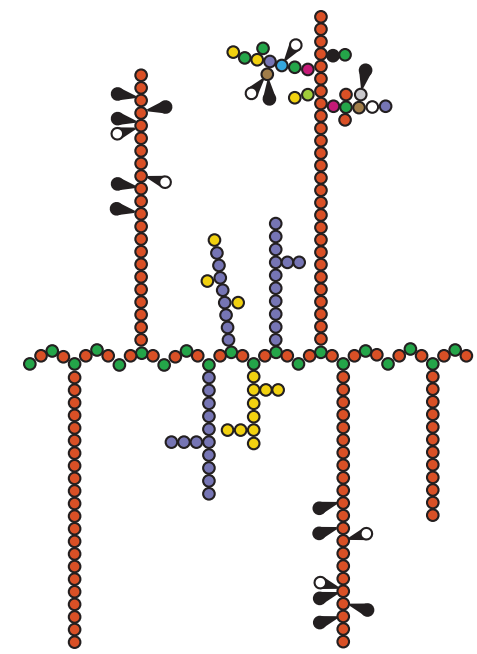

\begin{tabular}{|ll|}
\hline Acetil éster & Metil éster \\
○ Ácido galacturônico (GalA) & ○ Ramnose (Rha) \\
○ Apiose (Api) & ○ Fucose (Fuc) \\
O Ácido acérico (AceA) & ○ Galactose (Gal) \\
O Arabinose (Ara) & ○ Xilose (Xyl) \\
O Ácido glucurônico & Ácido cetodeoximano- \\
O Ácido deoxi-lixoheptulo & octulopiranosilônico (KDO) \\
piranosilárico (Dha) & \\
\hline
\end{tabular}

Alternativa proposta

Figura 1. Alternativa para representação da cadeia péctica. 
de reação, na Figura 2. O primeiro tipo deles é a desesterificação, com liberação de metanol e formação de pectatos. Em meio ácido ( $\mathrm{pH}$ entre 1 a 3 ), em temperaturas inferiores a $10{ }^{\circ} \mathrm{C}$, predominam as desesterificações. $\mathrm{O}$ outro mecanismo, denominado de despolimerização (diminuição do tamanho da cadeia polimérica) pode acontecer por hidrólise (ácida ou enzimática) das ligações $\alpha(1 \rightarrow 4)$, ou ainda por reações de $\beta$-eliminação. Em temperaturas acima de $10{ }^{\circ} \mathrm{C}$ em meio ácido, são mais frequentes as hidrólises de ligações $\alpha(1 \rightarrow 4)^{[40]}$. Na $\beta$-eliminação, o hidrogênio em C5, mais ácido em função do grupo éster metílico é atacado pelo íon hidróxido, resultando na transferência eletrônica e levando à ruptura da ligação glicosídica e à formação, entre C4 e C5, de dupla ligação conjugada com aquela da função carboxílica. Essa reação pode ser acelerada pelo aumento da concentração de álcali e da temperatura ou na presença de íons tais como $\mathrm{Ca}++, \mathrm{Mg}++, \mathrm{K}+, \mathrm{Cl}-$, citrato, malato ou fitato. Abaixo de $10{ }^{\circ} \mathrm{C}$, o grau de metilação das pectinas é suficientemente reduzido para impedir, por repulsão elestrostática, a aproximação dos íons hidróxidos e essa reação não mais ocorre ${ }^{[26]}$.

As substâncias pécticas são degradadas igualmente por enzimas sintetizadas por vegetais ou por microrganismos. Nesse caso, a degradação pode seguir duas vias principais: desmetilação preliminar pela pectina-esterase, seguida da hidrólise ácida das ligações $\alpha(1 \rightarrow 4)$ pelas poligalacturonases; despolimerização direta das pectinas por reações também de $\beta$-eliminação sob ação das pectinas liases e pectato liases de origem microbiana ${ }^{[40]}$.

As condições de extração apresentam importante influência sobre a natureza da pectina, tanto nos aspectos quantitativos quanto qualitativos, podendo ser modificadas ( $\mathrm{pH}$, temperatura, tempo) resultando em alterações no rendimento, na capacidade geleificante e no grau de esterificação (DE). A razão da matéria-prima e solvente deve também ser ajustada, visando a separação das fases sólida e líquida, a filtrabilidade do extrato e o custo da evaporação da água no processo. Dessa maneira, é possível controlar a extração de pectina para otimizar seu uso potencial ${ }^{[1,77]}$.

\section{Caracterização de Substâncias Pécticas}

Existem três fatores principais relacionados à sua natureza que dificultam o estudo das moléculas pécticas: caráter polimérico e presença de cargas elétricas, que influenciam a solubilidade, a degradação química, o comportamento hidrodinâmico em dispersão e iônico, na cromatografia; estrutura polissacarídica heteropolimérica, comportando uma gama variada de unidades estruturais, com diferentes substituintes, formas, anomerias e tipos de ligação, o que dificulta sua análise estrutural e problemas ligados à extração, associados à insolubilização dos polissacarídeos pelo tratamento para inativação enzimática e aos agentes de extração e protocolos de obtenção com pectinas de diferentes características ${ }^{[26]}$.

Um conhecimento detalhado da estrutura da pectina e de suas propriedades é necessário para assegurar um produto adequado a cada processo ${ }^{[21]}$. $\mathrm{O}$ estudo das substâncias pécticas surge como importante ferramenta para implementação de melhorias no processamento de frutas ${ }^{[78]}$. Entretanto, a determinação do conteúdo de pectina em alimentos mostra algumas dificuldades inerentes à estrutura das moléculas pécticas e interferências causadas por outros carboidratos nas amostras, sejam ácidos ou não ${ }^{[79]}$.

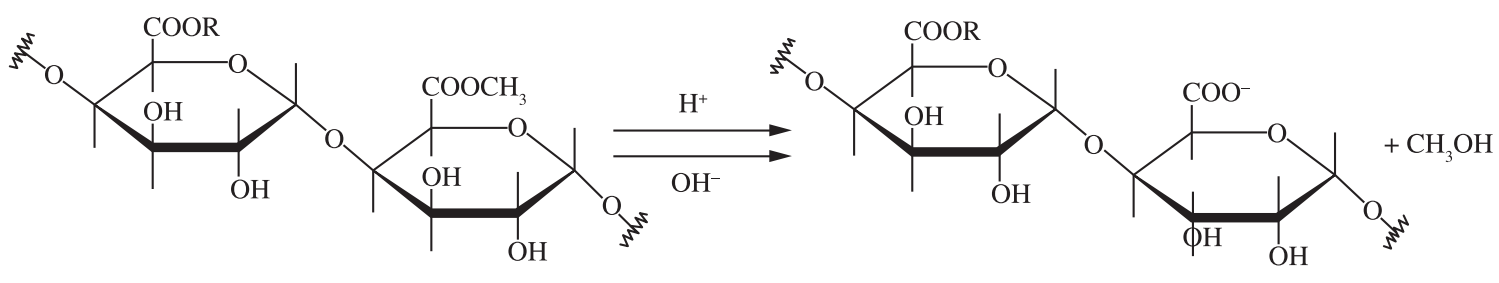

Desesterificação/saponificação

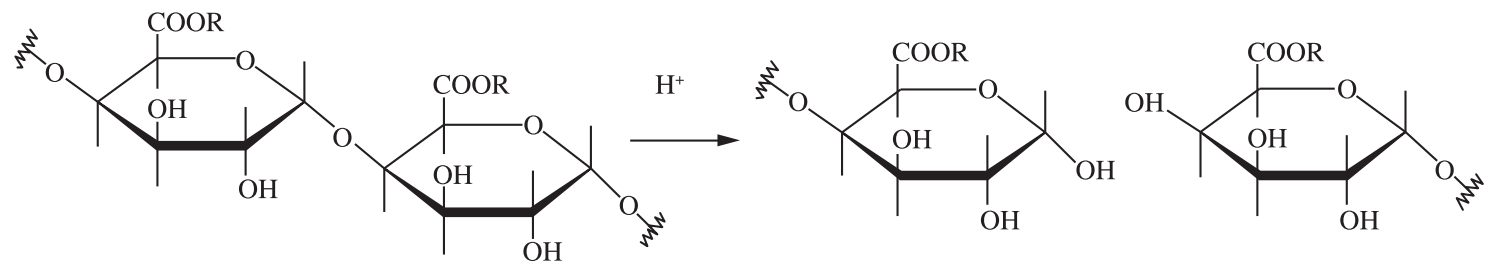

Hidrólise ácida

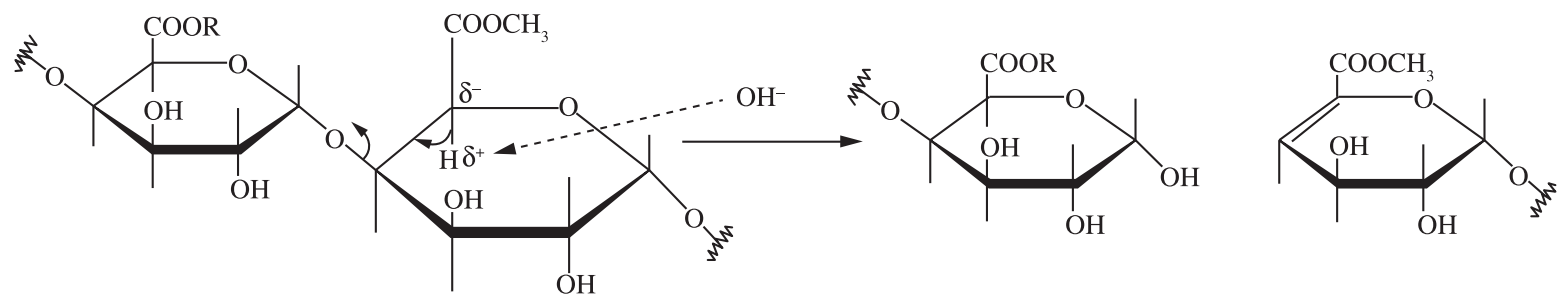

$\beta$-eliminação

Figura 2. Principais modificações nas substâncias pécticas por efeito de temperatura. 
Para a elucidação completa da estrutura molecular de um polisacarídeo é preciso determinar as seguintes características com relação às unidades monossacarídicas constituintes: espécie, configuração D ou L, número por molécula, forma do anel, sequência nas cadeias, bem como posição e configuração anomérica das ligações glicosídicas entre eles ${ }^{[80]}$. Todavia, para fins de aplicação tecnológica, esse grau de detalhamento não se faz necessário; há algumas técnicas analíticas usuais para caracterização qualitativa e quantitativa das substâncias pécticas, comentadas a seguir.

Para determinação de açúcares neutros, a cromatografia a gás (CG) é preferível à líquida de alta pressão (HPLC), devido a uma série de fatores. Além da sensibilidade, permitindo a análise de quantidades menores que nanomols de carboidratos, mostra-se menos susceptível a interferências, como de sais ou proteínas ${ }^{[81]}$. Pelo contrário, a HPLC apresenta a vantagem de permitir a quantificação dos ácidos urônicos juntamente com os açúcares neutros, se adicionada uma etapa de purificação para eliminação de contaminantes $^{[82]}$. O método mais usual para determinação de ácidos urônicos era a reação com carbazol, não específica para o ácido galacturônico. Posteriormente, o reativo foi substituído pelo metahidroxifenil, proposto em 1973 por Blumenkrantz e Asboe-Hansen. Esse método apresenta elevada sensibilidade e especificidade, com menor interferência por açúcares neutros como a glucose, a galactose, a xilose, a arabinose, a ramnose, a frutose ou componentes fenólicos ${ }^{[79,83]}$. A utilização de um tubo em branco para cada amostra analisada é uma das maneiras de se diminuir a interferência de cor promovida pela hidrólise de outros componentes, sendo o valor corrigido pela diferença da coloração da reação pela adição do reativo e da coloração resultante da hidrólise ${ }^{[84]}$. Entretanto, para eliminar o escurecimento ocasionado na presença de açúcares neutros, há um método proposto com adição de quantidades mínimas de sulfamato, sem significativa redução da sensibilidade na detecção de ácidos urônicos ${ }^{[85]}$.

Em todas as pectinas naturais, parte dos grupos carboxilas da cadeia principal de ácido galacturônico está esterificada, sendo os substituintes mais comuns os grupos metila ${ }^{[5]}$. Essa substituição é expressa como grau de esterificação (DE) ou grau de metilação (DM). Embora, na prática, sejam utilizados de forma indistinta, o DM corresponde ao percentual do total de resíduos de ácido galacturônico esterificados apenas com grupo metoxila ${ }^{[86]}$, enquanto o $\mathrm{DE}$, à razão dos grupamentos esterificados de ácido D-galacturônico pelo total de grupamentos de ácido galacturônico. O DE inclui outros grupos capazes de promover a esterificação além dos grupos metila, como os grupos acetila ${ }^{[5]}$. O valor global de esterificação é um importante fator de influência sobre a geleificação, associado com outras variáveis interdependentes, como a massa molar, heterogeneidade, $\mathrm{pH}$, grau de amidação, presença de açúcares e outros solutos ${ }^{[3,87]}$.

$\mathrm{O}$ percentual de grupos esterificados (DM ou DE) é mais comumente determinado por titulação ${ }^{[88]}$ ou ligação com cobre também usado para análise do teor de ácido galacturônico. Também têm sido utilizadas as espectroscopias de infravermelho (FTIR) ${ }^{[89-91]}$ e ressonância magnética nuclear $(\mathrm{RMN})^{[92]}$. Outra maneira de calcular é a partir dos conteúdos de grupamentos metoxil e ácido galacturônico. $\mathrm{O}$ metanol pode ser determinado colorimetricamente, por cromatografia a gás (GLC), como metil nitrito, por pirólise acoplada à GLC ou à espectrometria de massa ${ }^{[1]}$ e por HPLC ${ }^{[93]}$.

As pectinas em soluções diluídas apresentam comportamento de fluidos Newtonianos, mas, em concentrações moderadas, exibem comportamento não-Newtoniano ${ }^{[5]}$, resultando, nesse caso, em medidas de viscosidade aparente ${ }^{[94]}$. Em geral, as pectinas, longas cadeias macromoleculares, mostram comportamento pseudoplástico, independente do seu grau de metoxilação. Assim, o decréscimo da viscosidade com o aumento da taxa de cisalhamento torna-se mais importante quando a concentração do polímero e a massa molar aumentam. Para baixas concentrações, a viscosidade é intimamente independente da tensão de cisalhamento. O desenvolvimento de novas determinações reológicas ampliou as possibilidades de caracterização da pectina em soluções e géis. Essas fornecem dados importantes como o fluxo do processo tecnológico para controle de qualidade ou padronização de produtos e a determinação de dados específicos para cada pectina, na pesquisa e no desenvolvimento de produto $^{[95]}$.

Os polímeros modificam a reologia em virtude de apresentarem elevada massa molar, entrelaçamentos na cadeia polimérica e interação do polímero com o solvente. Sua influência na reologia resulta do grande volume das macromoléculas em solução, comparadas ao total das dimensões moleculares das unidades básicas constituintes ${ }^{[96]}$. A melhor representação da morfologia de uma partícula polimérica é a de um "novelo aleatório". Quanto maior a interação entre os segmentos do polímero e das moléculas do solvente, tanto maior será o novelo polimérico. A viscosidade está diretamente relacionada ao volume hidrodinâmico da partícula, dependendo da massa molar e dessa interação. Outra variação estrutural que os polímeros podem assumir é a ramificação. As relações usuais dos parâmetros viscosimétricos e a estrutura da partícula para o polímero disperso só são válidas para polímeros lineares ou com pequenas ramificações. A ramificação aumenta a densidade do novelo polimérico, sendo que um polímero ramificado ocupa um volume menor do que linear de mesmo tamanho e, consequentemente, apresenta uma viscosidade intrínseca menor ${ }^{[97]}$.

Os géis de pectina são comumente vistos como sistemas híbridos de duas fases, com alto grau de interface entre o sistema contínuo, sob a forma de rede tridimensional com longas cadeias de ácidos pectínicos e a fase aquosa aprisionada, igualmente contínua ou finamente dispersa. Em certos casos, especialmente nas pectinas de beterraba, as funções acetilas reduzem fortemente $o$ poder geleificante, sendo que $2,6 \%$ de acetilas já reduzem quase que completamente essa característica. Pelo contrário, o ácido péctico fortemente acetilado pode mostrar uma aptidão à geleificação em meio ácido açucarado. Assim, a geleificação, prejudicada por quantidades relativamente fracas de grupos acetilas, torna-se novamente possível quando uma forte acetilação recria uma nova superficie hidrofóbica de grupos acetilas ${ }^{[98]}$. A presença de amido e proteína pode contribuir para o aumento da viscosidade em frações pécticas analisadas ${ }^{[99]}$.

Em numerosos casos, uma estreita relação foi observada entre o poder geleificante e o grau de polimerização, bem como a viscosidade intrínseca. Certos fatores alteram a relação entre a massa molar e o poder geleificante, como a presença de cadeias ou grupos laterais, que ocultam os grupos funcionais necessários para a geleificação ${ }^{[98]}$. O padrão de distribuição das cadeias laterais influencia a geleificação das pectinas ${ }^{[3]}$.

A massa molar das pectinas pode ser variável de acordo com a fonte vegetal, matéria-prima e condições de extração, mas sua determinação é um desafio devido aos problemas de heterogeneidade e agregação, além da usual larga distribuição ${ }^{[1,27]}$. Comparativamente, os valores de massa molar de pectinas são notadamente menos elevados que os da celulose ou do amido. Os valores citados na literatura variam entre 20.000 a 360.000 g.mol ${ }^{-1}$ e as amostras comerciais entre 35.000 a 120.000 g.mol ${ }^{-1}$, segundo o tipo de pectina. Em função da polimolecularidade das substâncias pécticas, os valores médios podem diferir até de um fator de 10 . Além dos fatores já citados, essa importante variação pode ser explicada pela utilização de métodos diferentes de extração ou determinação da massa molar ${ }^{[28]}$. 
Os cálculos da forma e tamanho molecular a partir da viscosidade são válidos somente se a medida não for influenciada por cargas e efeitos de agregação. Sendo uma molécula com regiões de diferente polaridade, os efeitos de carga são consideráveis. A massa molar calculada a partir dos dados de viscosidade intrínseca é provavelmente imprecisa por vários fatores, possíveis de interferir na tensão superficial como hidratação, flexibilidade da molécula e heterogeneidade ${ }^{[11]}$. A massa molar média é calculada por meio de medidas de viscosidade. Em muitos casos, grandes discrepâncias irão acarretar o mesmo efeito na massa molar média, mesmo quando os experimentos são conduzidos com especial cuidado. Isso pode ser devido a fatores como 1-polidispersão; 2- fenômeno de agregação dependente do DE e concentração que interferem na coleta dos dados por espalhamento de luz e 3-dificuldades experimentais durante a titulação dos grupamentos finais, especialmente para amostras com elevada massa molar ${ }^{[1]}$.

A técnica de exclusão estérica em gel tornou-se um método popular para determinação do tamanho molecular bem como da homogeneidade. Por meio de polissacarídeos de tamanho molecular conhecido, utilizados como marcadores, a massa molar pode ser determinada. Um pico simétrico de eluição é indicativo de homogeneidade ${ }^{[80]}$.

\section{Aplicações da Pectina}

No setor industrial, os polissacarídeos pécticos promovem aumento de viscosidade e funcionam como coloide estabilizante e protetor em alimentos e bebidas, com aplicação em doces e geleias, preparações de frutas para iogurtes, bebidas e sucos de frutas concentrados, sobremesas de frutas e leite, produtos lácteos gelificados, produtos de confeitaria e produtos lácteos acidificados diretamente ou fermentados. Dentre outras propriedades estão a prevenção de flotação em preparados de frutas, a estabilidade de produtos de panificação, a estabilização proteica, a maciez a partir da melhoria da textura, o aumento do volume e o controle da sinerese ${ }^{[4]}$.

Com relação aos efeitos farmacológicos da pectina, considera-se que no grande grupo das fibras dietéticas, integra o grupo dos polissacarídeos não-amiláceos, juntamente com gomas, hemiceluloses, $\beta$-glucanas, entre outros. Embora esses compostos não sejam degradados por enzimas humanas, podem ser pela microbiota natural, especialmente durante a passagem pelo intestino grosso ${ }^{[100]}$. Com implicações benéficas do equilíbrio hídrico e motilidade intestinal, as substâncias pécticas são inadequadamente denominadas de fibras solúveis, visto que em solução são encontradas sob a forma de dispersão coloidal ${ }^{[101,102]}$. A capacidade de formação de géis pela pectina está relacionada à quantidade de grupos polares livres (como as hidroxilas), à estrutura tridimensional, ao $\mathrm{pH}$, aos eletrólitos presentes no meio, à solubilidade e concentração da pectina na dispersão, à massa molar, ao grau de metilação, à natureza e à qualidade da fruta a ser geleificada, à tecnologia (procedimento, material de cozimento e temperatura, entre outros), à distribuição dos grupamentos ao longo da cadeia péctica e à composição das cadeias laterais ${ }^{[26,40,103]}$. Com efeitos prébióticos, a cadeia péctica pode ser transformada em ácidos graxos de cadeia curta (acético, butírico e propiônico), bem como em dióxido de carbono, pela ação de bactérias produtoras de enzimas pectinolíticas dos gêneros Aerobacillus, Lactobacillus, Micrococcus e Enterococcus. Assim, a pectina apresenta fraca tendência laxativa e estimula o crescimento da microbiota no cólon $^{[104]}$. Existem outras propriedades de promoção à saúde, comprovadas cientificamente, associadas às substâncias pécticas. Dentre essas, podem ser destacadas: 1-redução do colesterol total pelo decréscimo da absorção do colesterol exógeno, em função do caráter hidrofóbico dos grupamentos metiléster; 2-ligação com produtos de degradação no cólon, aumento da excreção de ácidos biliares e redução da reabsorção de ácidos biliares no intestino e no fígado; 3-diminuição das frações popularmente conhecidas como mau colesterol (LDL) e embora não altere o bom colesterol (HDL), também pode ser protetora contra a aterosclerose por melhorar a razão HDL/LDL; 4-aumento da viscosidade do líquor da digestão e da espessura da camada da parede intestinal interna, reduzindo a absorção de glucose; 5-redução do peso corporal pela imobilização de nutrientes nos intestinos, aumento da sensação de saciedade e diminuição da atividade de certas enzimas, que leva à menor digestão e absorção; 6-ligação a metais pesados e a microrganismos tóxicos no cólon impedindo a reabsorção das toxinas por estes produzidas $^{[15,105]}$.

Com relação aos possíveis efeitos negativos, há estudos indicando a ligação de pectinas de baixa metoxilação com cátions bivalentes, o que influenciaria negativamente a absorção de zinco. Isso, porém, é contraditório, visto que a degradação da pectina ocorre no cólon, e esse mineral ainda pode ser absorvido nessa porção do intestino, após a hidrólise ${ }^{[103]}$. Como uso não alimentício, a pectina serve à produção de uma variedade de produtos especiais, incluindo filmes biodegradáveis e comestíveis, adesivos, substitutos de papel, espumas e plastificantes, agentes de superfície para produtos médicos, materiais para implantes biomédicos e liberação de fármacos ${ }^{[58]}$. A pectina, em função de suas características estruturais e propriedades reológicas, parece ter potencial na elaboração de formulações farmacêuticas ${ }^{[106]}$, com estudos de cinética e hidratação, para administração por via oral ${ }^{[107]}$ ou aplicação tópica ${ }^{[108]}$, revestimento de sistemas sólidos orais ${ }^{[109]} \mathrm{e}$ liberação de fármacos in situ com mínima degradação ${ }^{[110]}$.

\section{Conclusão}

Para produção de pectina em escala industrial, o empirismo ainda permanece como bom senso, sem controle rigoroso dos fatores de produção durante o processamento com a padronização posterior pela mistura de diferentes lotes de pectina, também com outras substâncias. Assim, a obtenção de uma pectina de qualidade reológica elevada pode favorecer o custo/benefício no balanço final para obtenção de uma pectina comercial, um produto padronizado que visa assegurar aos clientes a força do gel descrita e desejada.

Embora os estudos sobre substâncias pécticas não sejam recentes, as publicações sobre o assunto ainda são escassas no Brasil. A pesquisa sobre esse polímero mostra-se promissora, com necessidade de estudos aprofundados sobre a matéria-prima, o processo de extração e a qualidade do produto final, com ênfase nas propriedades reológicas dos polissacarídeos obtidos.

\section{Agradecimentos}

Os autores agradecem a CAPES, UFPR, GTM-UEPG e UTFPR pelo apoio.

\section{Referências Bibliográficas}

1. Voragen, G. J.; Pilnik, W.; Thibault, J. F.; Axelos, M. A. V. \& Renard, C. M. G. C-"Pectins", in: Food polysaccharides and their applications, cap. 10, Stephen A. M. (ed.), Marcel Dekker Inc., New York (1995).

2. Pérez, S.; Rodríguez-Carvajal, M. A. \& Doco, P.-Bioch., 85, p.109 (2003).

3. Willats, W. G. T; Knox, J. P. \& Mikkelsen, J. D.- Trends Food Sc. Tech., 17, p.97 (2006). PMid:21299575. http://dx.doi.org/10.1016/j. tifs. 2005.10 .008

4. Voragen, A. G. J.; Coenen, G.-J.; Verhoef, R. P. \& Schols, H. A.-Struct. Chem, 20, p.263 (2009). http://dx.doi.org/10.1007/s11224-009-9442-Z

5. Sriamornsak, P. - Silpak. Univ. Int. J., 3, p.206 (2003). 
6. Office Federal de la Sante Publique-OFSP.-"Gèlifiants et èpaississants" (2005). Disponível em: <http://www.bag-anw.admin.ch/SLMB_Online_ PDF/Data\%20SLMB_MSDA/Version\%20F/40_Gelifiants.pdf>.

7. Kertesz, Z. I.-“The pectic substances", Interscience, New York (1951).

8. Braverman, J. B. S. \& Berk, Z.-"Braverman's Introduction to the Biochemistry of Foods", Elsevier, New York (1976).

9. Joslin, M. A. \& Deuel, H.- J. Food Sc., 28, p.65 (1963). http://dx.doi. org/10.1111/j.1365-2621.1963.tb00161.x

10. Nogueira, A.; Wosiacki, G.; Falcão, L. D. \& Chiquetto, N. C. Associação Gaúcha dos Produtores de Maçã-AGAPOMI. (2008). Disponível em: <http:// www.agapomi.com.br/jornal.php?noticia=50>.

11. Owens, H. S.; Lotzkar, H.; Schultz, T. H. \& Maclay, W. D. - J. Am. Chem. Soc., 08, p.1628 (1946). PMid:20995001. http://dx.doi. org/10.1021/ja01212a079

12. Baker, G. "High polymer pectins and their deesterification", in: Advances in food research, Mrak, E. M. \& Stewart, G. F. (ed.), Academic Press, New York (1948).

13. Cybercolloids. (2008). Disponível em: <http://www.cybercolloids.net/ library/pectin/introduction.php>.

14. Elwell, W. E. \& Dehnt, W. M.- Plant Phys., 14, p.809 (1939). PMid:16653603. PMCid:437789. http://dx.doi.org/10.1104/pp.14.4.809

15. Internacional Pectin Producers Association - IPPA.-"What is Pectin?" (2006). Disponível em: <http://www.ippa.info/what_is_pectin.htm>.

16. Herbstreith \& Fox. (2009). Disponível em: <http://www.herbstreithfox.de/fileadmin/tmpl/pdf/funde/FuE_Pectins_in_Preventive_ Nutrition_and_Therapy.pdf>.

17. Ribeiro, B. M.-“Visão geral da empresa CPKelco", Reunião Técnica, Limeira 2002.

18. Associação Comercial e Industrial de Limeira-ACIL. (2010). Disponível em: <http://www.acil.org.br/site/diadaempresa/2007/ encarte $01 /$ jornal10.htm $>$.

19. Schieber, A.; Hilt, P.; Streker, P.; Endress, H.-U.; Rentschler, C. \& Carle, R.-Inn. Food Sc. Emerg. Tech., 04, p.99 (2003). http://dx.doi. org/10.1016/S1466-8564(02)00087-5

20. Bates, R. P.; Morris, J. R. \& Crandal, P. G. Agricult. Serv. Bul. FAO, 146, p.25 (2001).

21. Intermediate Technology Development Group-ITDG.-"Fruit Waste Utilisation" (2007). Disponível em: <http://www.itdg. org/docs/technical_information_service/fruit_waste_utilisation. pdf\#search=\%22wastes\%20fruits\%22>.

22. Cybercolloids. (2010). Disponível em: <http://www.cybercolloids.net/ manufacturers/list.php?submit=go\&gum=pectin $>$.

23. Yalpani, M. \& Sandford, P. A. "Commercial Polysaccharides: recent trends and developments", in: Industrial polysaccharides. genetic engineering, structure/property relations and applications, Yalpani, M. (ed.) Elsevier, Amsterdan (1987).

24. Quimibras. - "Lista de Preços e Produtos" (2011). Disponível em: $<$ http://www.quimibras.com.br/homepage/produtos/dyne_todos.html>.

25. Christensen, S. H. - "Pectins", in: Food Hydrocolloids, Glicksman M. (ed.), CRC Press, Florida (1984).

26. Thibault , J. F.; Saulnier, L.; Axelos, M. A. V \& Renard, C. M. G. C. Bull. Soc. Bot. Fr., 314, p.319 (1991).

27. Sakai, T.; Sakamoto, T.; Hallaert, J. \& Vandamme, E.-Adv. Appl. Micr., 39, p.213 (1993). http://dx.doi.org/10.1016/S0065-2164(08)70597-5

28. Thibault, J. F.-“Les substances pectiques", in: Les polymeres vegetaux, Monties B. (ed.), Gaulthier-Vilars, Paris (1980).

29. Daniells, S. - "Pectin: Sourcing Issues and Ideas" (2008). Disponível em: $<$ http://www.foodnavigator.com/Science-Nutrition/Pectin-sourcingadvances-2007>.

30. Mesbahi, G.; Jamalian, J. \& Farahnaky, A. - Food Hydr., 19, p.731 (2005). http://dx.doi.org/10.1016/j.foodhyd.2004.08.002

31. Iglesias, M. T. \& Lozano, J. E. J. - Food Eng., 62, p.215 (2004). http:// dx.doi.org/10.1016/S0260-8774(03)00234-6
32. Faravash, R. S. \& Ashtiani, F. Z. - Food Hydr., 22, p.196 (2008). http:// dx.doi.org/10.1016/j.foodhyd.2007.04.003

33. Fertonani, H. C. R.; Scabio, A.; Schemin, M. H. C.; Carneiro, E. B. B; Nogueira, A. \& Wosiacki, G. - Semina: C. Agr., 27, p.599 (2006).

34. Scabio, A.; Fertonani, H. C. R.; Schemin, M. H. C. Petkowicz, C. L. O.; Carneiro, E. B. B.; Nogueira, A. \& Wosiacki, G. - Braz. J. Food Tech., 10, p.259 (2007).

35. Yapo, B. M.; Robert, C.; Etienne, I.; Wathelet, B. \& Paquot, M. - Food Chem., 100, p.1356 (2007). http://dx.doi.org/10.1016/j. foodchem.2005.12.012

36. Yapo B. M.; Lerouge P.; Thibault J. F. \& Ralet, M. C. - Carb. Pol., 69, p.426 (2007). http://dx.doi.org/10.1016/j.carbpol.2006.12.024

37. Tamaki, Y.; Konishi, T.; Fukuta, M.; Tako, M. - Food Chem., 107, p.352 (2008). http://dx.doi.org/10.1016/j.foodchem.2007.08.027

38. Fertonani, H. C. R.; Scabio, A.; Carneiro, E. B. B.; Schemin, M. H.C.; Nogueira, A. \& Wosiacki G. - Braz. Arch. Biol. Tech., 52, p.177 (2009). http://dx.doi.org/10.1590/S1516-89132009000100023

39. Thomas, M.; Guillemin, F.; Guillon, F. \& Thibault, J.F. - Carb. Pol., 53, p.361 (2003). http://dx.doi.org/10.1016/S0144-8617(03)00118-8

40. Singthong, J.; Cui, S. W.; Ningsanond, S. \& Goff, H. D. - Carb. Pol., 58, p.391 (2004). http://dx.doi.org/10.1016/j.carbpol.2004.07.018

41. Singthong, J.; Ningsanond, S.; Cui, S. W. \& Goff, H. D. - Food Hydr., 19, p.793 (2005). http://dx.doi.org/10.1016/j.foodhyd.2004.09.007

42. Koubala, B. B., Mbome, L. I., Kansci, G., Tchouanguep, M. F., Crepeau, M. J., Thibault, J. F. \& Ralet, M. C. - Food Chem., 106, p.1202 (2008). http://dx.doi.org/10.1016/j.foodchem.2007.07.065

43. Koubala, B. B.; Kansci, G., Mbome, L. I., Crepeau, M. J., Thibault, J. F. \& Ralet, M. C. - Food Hydr., 22, p.1345 (2008). http://dx.doi. org/10.1016/j.foodhyd.2007.07.005

44. Sirisakulwat, S.; Nagel, A.; Sruamsiri, P., Carle, R. \& Neidhart, S. J. Agric. Food Chem., 56, p.10727 (2008). PMid:18980323. http:// dx.doi.org/10.1021/jf802173c

45. Sirisakulwat, S.; Sruamsiri, P.; Carle, R. \& Neidhart, S. - Int. J. Food Sc. Tec., 45, p.1647 (2010). http://dx.doi.org/10.1111/j.1365-2621.2010.02314.x

46. Westereng, B.; Michaelsen, T.E., Samuelsen, A. B. \& Knutsen, S. H. - Carb. Pol., 72, p.32 (2008). http://dx.doi.org/10.1016/j. carbpol.2007.07.017

47. Emaga, T. H.; Happi, T.; Ronkart, S. N.; Robert, C.; Wathelet, B. \& Paquot, M. - Food Chem., 108, p.463 (2008).

48. Mollea, C.; Chiampo, F. \& Conti, R. - Food Chem., 107, p. 1353 (2008).

49. D’Addosio, R. D.; Páez, G., Marín, M , Mármol, Z. \& Ferrer, J. - Rev. Fac. Agr. LUZ, 22, p.241 (2005).

50. Yapo, B. M. \& Koffi, K. L. - J. Agr. Food Chem., 54, p.2738 (2006). PMid:16569069. http://dx.doi.org/10.1021/jf052605q

51. Yapo, B. M. \& Koffi, K. L. - J. Sc. Food Agr., 88, p.2125 (2008). http:// dx.doi.org/10.1002/jsfa.3323

52. Yapo, B. M. - J. Agr. Food Chem., 57, p.1572 (2009). PMid:19199593. http://dx.doi.org/10.1021/jf802969m

53. Yapo, B. M. - Food Res. Int., 42, p.1197 (2009). http://dx.doi. org/10.1016/j.foodres.2009.06.002

54. Canteri, M. H. G.; Scheer, A. P.; Ginies C.; Renard C. M. G. C. \& Wosiacki G. - Rev. Bras. Tec. Ag., 04, p.109 (2010).

55. Thakur, B. R.; Singh, R. K. \& Handa, A. K. - Crit. Rev. Food Sc. Nut., 37, p.47 (1997). PMid:9067088. http://dx.doi.org/10.1080/10408399709527767

56. Thibault, J. F. \& Petit, R. - Ind. Alim. Agr., 96 (1979).

57. Carpita, N.; McCann, M. - "The cell wall" in: Buchanan, B. B.; Gruissem, W. \& Jones, R. L. (eds.), American Society of Plants Physiologists, Berkeley (2000).

58. Mohnen, D. - Cur. Op. Plant Biol., 11, p.266 (2008).

59. Vincken, J. P., Schols, H. A.; Oomen, R. J. F. J.; McCann, M. C.; Ulvskov, P., Voragen, A. G. J. \& Visser, R. G. S. - Plant Phys., 132, p.1781 (2003). PMid:12913136. PMCid:1540329. http://dx.doi. org/10.1104/pp.103.022350 
60. Somerville, C.; Bauer, S.; Brininstool, G.; Facette, M.; Hamann, T.; Milne, J.; Osborne, E.; Paredez, A.; Persson, S.; Raab, T.; Vorwerk, S. \& Youngs, H. - Science, 306, p.2206 (2004). PMid:15618507. http:// dx.doi.org/10.1126/science.1102765

61. Hahn, M. G.; Darvill, A. G. \& Albersheim, P. - Plant Physiol., 68, p.1161 (1981). PMid:16662068. PMCid:426062. http://dx.doi. org/10.1104/pp.68.5.1161

62. Huber, D. J.; Karakurt, Y. \& Jeong, J. - R. Bras. Fisiol. Veg., 13, p.224 (2001). http://dx.doi.org/10.1590/S0103-31312001000200009

63. Ridley, B. L.; O’Neil, M. A. \& Mohnen, D. - Phytochem., 57, p.929 (2001). http://dx.doi.org/10.1016/S0031-9422(01)00113-3

64. Santos, M. S.; Carneiro, P. I. B.; Wosiacki, G.; Petkowicz, C. L. O. \& Carneiro, E. B. B. -Semina: C. Agr., 30, p.101 (2009).

65. Santos, M. S.; Petkowicz, C. L. O.; Haminiuk, C. W. I. \& Candido, L. M. - Rev. Iberoam. Polim., 11, p.359 (2010).

66. Santos, M. S.; Petkowicz, C. L. O.; Haminiuk, C. W. I. \& Candido, L.M. B. - Polímeros, 20, p.352 (2010).

67. Antunes, L. E. C.; Gonçalves, E. D. \& Trevisan R. - R. Bras. Agroc., 12, p.57 (2006).

68. Haminiuk, C. W. I.; Sierakowski, M.-R.; Izidoro, D. R.; Maciel, G. M.; Scheer, A. P. \& Masson, M. L. - Ciênc. Tecnol. Aliment., 29, p.225 (2009). http://dx.doi.org/10.1590/S0101-20612009000100035

69. Oliveira, M. N. S.; Gusmão, E.; Lopes, P. S. N.; Simões, M. O. M.; Ribeiro, L. M.; Dias, L. M. \& Dias, B. A. S. - Rev. Bras. Frutic., 28, p.380 (2006). http://dx.doi.org/10.1590/S0100-29452006000300010

70. Pinheiro, A. C. M.; Vilas Boas E. C. B. \& Lima L. C. - Ciênc. Tecnol. Aliment., 25, p.32 (2005). http://dx.doi.org/10.1590/S010120612005000100006

71. Kliemann, E.; Simas, K. N.; Amante, E. R.; Prudêncio, E. S.; Teófilo, R. F.; Ferreira, M. M. C. \& Amboni, R. M. D. C. - Int. J. Food Sc. Technol., 44, p.476 (2009). http://dx.doi.org/10.1111/j.1365-2621.2008.01753.x

72. Pinheiro, E. R.; Silva, I. M. D. A.; Gonzaga, L. V.; Amante, E. R.; Teófilo, R. F.; R. F.; Ferreira, M. M. C. \& Amboni, R. M. D. C. - Bior. Technol., 99, p.5561 (2008).

73. Munhoz, C. L.; Sanjinez-Argandoña, E. J. \& Soares-Júnior, M. S. Ciênc. Tecnol. Aliment., 30, p.119 (2010). http://dx.doi.org/10.1590/ S0101-20612010005000013

74. Marcon, M. V.; Vriesmann, L. C.; Wosiacki, G.; Carneiro, E. B. \& Petkowicz, C. L. O. - Polímeros, 15, p.127 (2005).

75. Canteri-Schemin, M. H.; Fertonani, H. C. R.; Waszczynskyj, N. \& Wosiacki, G. - Braz. Arch. Biol. Technol., 48, p.259 (2005). http:// dx.doi.org/10.1590/S1516-89132005000200013

76. Sato, M. F.; Vieira, R. G.; Zardo, D. M.; Falcão, L. D.; Nogueira, A. \& Wosiacki, G. - Acta Scient. Agr., 32, p. 29 (2010).

77. Levigne, S.; Ralet, M.-C. \& Thibault, J. F. - Carb.Pol., 49, p.145 (2002). http://dx.doi.org/10.1016/S0144-8617(01)00314-9

78. Legentil, A.; Guichard, I.; Piffaut, B. \& Haluk, J. P. - Lebensm.-Wiss. U.-Tecnol., 28, p.569 (1995).

79. Ibarz, A.; Págan, A.; Tribaldo, F. \& Págan, J. - Food control, 17, p.890 (2006). http://dx.doi.org/10.1016/j.foodcont.2005.06.007

80. Pazur, J. H. - "Neutral polysaccharides", in: Carbohydrate analysis. Chaplin, M. F. \& Kennedy, J. F. (ed.), Oxford University Press, London, p.73-124 (1994).

81. Chaplin, M. F. - "Monosaccharides", in: Carbohydrate analysis. Chaplin, M. F. \& Kennedy, J. F.(ed.), Oxford University Press, London, p.01-41 (1994).

82. Leitão, M. C. A., Silva, M. L. A., Januário, M. I. N. \& Azinheira, H. G. - Carb. Pol., 26, p.165 (1995). http://dx.doi.org/10.1016/01448617(95)00003-P

83. Doner, L. W. - "Analytical methods for determining pectin composition", in: Chemistry and function of pectin. Fishmann, M. L. \& Jen, J. J. (eds.). ACS, Florida, p.13-19 (1986). http://dx.doi. org/10.1021/bk-1986-0310.ch002
84. Institute National de la Recherche Agronomique - INRA. - «UMR 408: Dosage acide galacturonique colorimétrie-01» (2009).

85. Filisetti-Cozzi, T. M. C. C. \& Carpita, N. C. - Anal. Biochem., 197, p.157 (1991).

86. Huismann, M. M. H.; Oosterveld, A. \& Schols, H. A. - Food Hydr., 18, p.665 (2004).

87. Bemiller, J. N. "Pectin". in: Chemistry and function of pectin. Fishmann, M. L. \& Jen, J. J. (eds.) ACS, Florida (1986).

88. Bochek, A. M.; Zabivalova, N. M. \& Petropavlosvskii, G. A. - Rus. J. App. Chem., 74, p.775 (2001).

89. Cerná, M.; Barros, A.; Nunes, A.; Rocha, S. M.; Delgadillo, I., Copíková, J. C. \& Coimbra, M. A. - Carb. Pol., 51, p.383 (2003).

90. Synytsya, A.; Copíková, J. C.; Matejka, P. \& Machovic, V. - Carb. Pol., 54, p.97 (2003). http://dx.doi.org/10.1016/S0144-8617(03)00158-9

91. Monsoor, M. A. - Carb. Pol., 61, p.362 (2005). http://dx.doi. org/10.1016/j.carbpol.2005.06.009

92. Renard, C. M. G. C. \& Jarvis, M. C. - Carb. Pol., 39, p.201 (1999). http://dx.doi.org/10.1016/S0144-8617(99)00006-5

93. Levigne, S., Thomas, M., Ralet, M. C., Quemener, B. \& Thibault, J. F. Food Hydr., 16, p.547 (2002). http://dx.doi.org/10.1016/S0268005X(02)00015-2

94. Hand, D. B.; Moyer, J. C.; Ransford, J. R.; Hening, J. C. \& Whittenberger, R. T. - Food Technol., 9, p.228 (1955).

95. Herbstreith \& Fox. - "Rheological Methods to Characterize Pectins in Solutions and Gels" (2008). Disponível em: <http://www.herbstreithfox.de/fileadmin/tmpl/pdf/funde/FuE_Rheological_Methods.pdf>.

96. Glass, J. E.; Schulz, D. N. \& Zuloski, C. F. - "Polymers as Rheology Modifiers", ACS, Washington (1991).

97. Pinto, M. R. - "Viscosidade de soluções diluídas de polímeros" (2010). Disponível em: <http://pcserver.iqm.unicamp.br/ mgo/cursos/qf732/ viscosidadepolimericas.pdf $>$.

98. Bondu, M. - "Proprietes fonctionnelles des pectines», in: CPCIA Proprietes fonctionelles des polysaccharides, Nantes, França (1986).

99. Iagher, F.; Reicher, F. \& Ganter, J. L. M. S. - Int. J. Biol. Macromol., 31, p.9 (2002). http://dx.doi.org/10.1016/S0141-8130(02)00044-2

100. Brody, T. - "Nutritional Biochemistry", Academic Press, London (1994).

101. Jafelicci Junior, M. \& Varanda, L. C. - Quím. nova esc., 9, p.20 (1999).

102. Wosiacki, G. \& Nogueira, A. - "Suco de maçâa", in: Venturini Filho, W. G. (ed.), Edgard Blücher, São Paulo, p.255-291 (2005).

103. Filisetti, T. M. C. C. \& Lobo, A. R. - "Fibra alimentar e seu efeito na biodisponibilidade de minerais", in: Cozzolino S. M. F.(ed.), Manole, São Paulo, p.175-215 (2007).

104. Mattes, F. - "Cholesterol and the Power of Pectin" (2009). Disponível em: <http://www.herbstreith-fox.de/fileadmin/tmpl/pdf/awtinfo/ AWT_Cholesterol_and_the_Power_of_Pectin.pdf $>$.

105. Endress, H. U. - "Nonfood uses of pectin", in: Walter R. \& Taylor, S. (ed.), Academic Press, New York (1991).

106. Villanova, J. C. O.; Oréfce R. L. \& Cunha, A. S. - Polímeros, 20, p.51 (2010).

107. Guimarães, G. G.; Katsuki, G. I.; Zanardo, N. D.; Ribeiro, D. A. \& Cavalcanti O. A. - Braz. J. Pharm. Sc., 44, p.133 (2008).

108. Cação, M.; Fernandes, F. B. \& Chorilli, M. - Rev. Bras. Farm., 90, p.272 (2009).

109. Souto-Maior, J. P. A.; Reis, A. V.; Pedreiro, L. N.; Cavalcanti, O. A. Braz. J. Pharm. Sc., 44, p.203 (2008).

110. Wong, T. W.; Colombo, G. \& Sonvico, F. - AAPS Pharm. Sci. Tech. (2010).

Enviado: 04/04/10

Reenviado: 11/07/11

Aceito: 19/07/11 